\title{
Transient Domain Structure of Coalescence-Induced Self-Organization in Droplet Phase Separation
}

\author{
C. M. Chou' ${ }^{1}$, W. T. Chuang ${ }^{1}$, P. D. Hong ${ }^{2}$ \\ ${ }^{1}$ National Synchrotron Radiation Research Center, Hsinchu, 30076, Taiwan, ${ }^{2}$ Department of Materials Science and Engineering, \\ National Taiwan University of Science and Technology, Taipei, 10607, Taiwan
}

chou.cm@nsrrc.org.tw

The picture of the phase separation constitutes a continuing source of inspiration for the development of multicomponent functional materials [1]. A key point of many applications is to precisely control the size and the connectivity of the phase-separated domain to achieve the desired structure and properties. However, the region of the forming the interconnected domains does not coincide with the spinodal curve, as often supposed, but that its region is actually much narrower, instead a densely packed droplet structure forms, i.e., the so-called "droplet spinodal decomposition (DSD)" [2]. A richness of kinetic phenomena was observed in the DSD, especially the hydrodynamic motion and collision of the droplets which are different from the pure diffusion in the NG and also deserve particular attention in the theoretical investigation. Obviously, an effective control of the DSD structure is much more complicated, because, in addition to some relevant material and thermophysical parameters, we also need to know the structural details, such as the droplet size, their size distribution, and the spatial correlation between them, at any instant of the evolution.

In this work, since the scatterers are known to be a collection of spherical droplets, our aim is to construct a suitable scattering function for the DSD structure and then to solve the droplet size distribution in real space by the indirect Fourier transformation (IFT) method [3]. Furthermore, unlike the most commonly used a priori strategies, we adopt a posteriori judgment to solve the IFT problems and yield quantitatively accurate descriptions of the transient domain structure in the bulk, especially in the droplet-size distribution, the well-defined short-range order, and the stress-optical phenomenon. Furthermore, the microscopic observation shows that at high droplet densities, the droplet collision and coalescence trigger a series of chain collisions which looks like a "ripple" propagating. The IFT results of the scattering by bulk specimen also support the observation. It is interesting that this new hydrodynamic phenomenon seems to be a nonequilibrium self-organization process and can occur only if the size of the coalescing droplets is greater than a threshold value.

(a) Quasi-2D
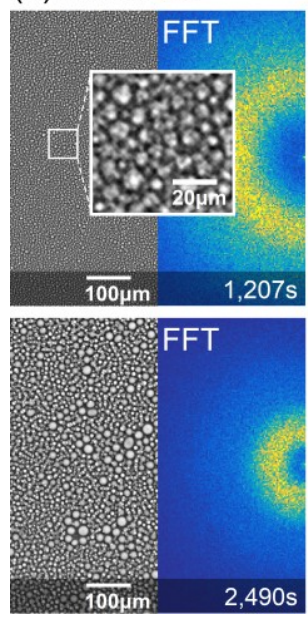

(b) Bulk
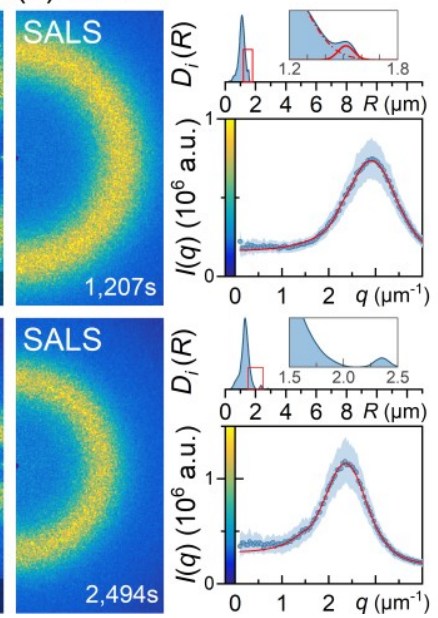
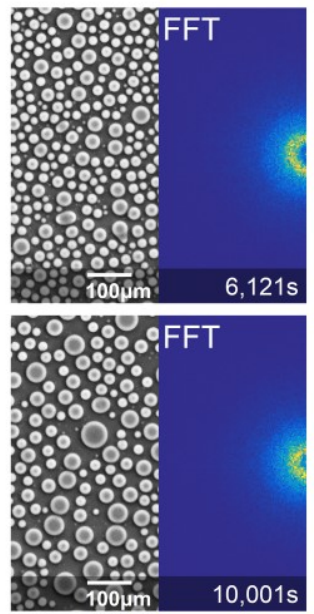
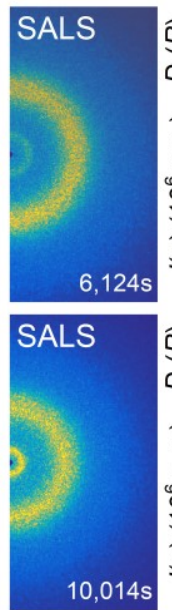
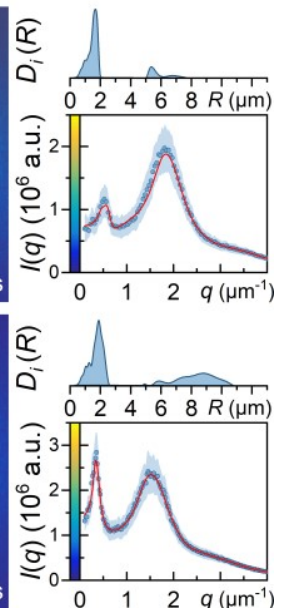

Figure 1. (a) Morphologies evolution of the DSD for the $\phi=0.54$ PEG/RAN mixture at $363 \mathrm{~K}$ in the $10 \mu \mathrm{m}$ sample thickness. (b) The evolution snapshots of the bulk DSD structure at different moments by the indirect Fourier transformation analysis.

[1] Wang, F., Altschuh, P., Ratke, L., Zhang, H., Selzer, M. \& Nestler, B. (2019). Adv. Mater. 31, 1806733.

[2] Shimizu, R. \& Tanaka, H. (2015). Nat. Commun., 6, 7407.

[3] Glatter, O. Scattering Methods and Their Application in Colloid and Interface Science (Elsevier, 2018).

Keywords: small-angle scattering; inverse scattering problem; spinodal decomposition; droplets Acta Cryst. (2021), A77, C579 